Schmerz 2021 · 35:2-4

https://doi.org/10.1007/s00482-021-00529-6

Angenommen: 4. Januar 2021

(c) Springer Medizin Verlag GmbH, ein Teil von Springer Nature 2021

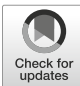

\section{Esther Pogatzki-Zahn ${ }^{1}$. Claudia Sommer ${ }^{2}$}

'Klinik für Anästhesiologie, operative Intensivmedizin und Schmerztherapie, Universitätsklinikum Münster, Münster, Deutschland

${ }^{2}$ Neurologische Klinik, Universität Würzburg, Würzburg, Deutschland

\title{
Prävention von Schmerzen - ein facettenreiches Global-Year- Thema 2020 mit viel Potenzial für Forschung und klinische Praxis
}

Die IASP (International Association for the Study of Pain) hat im Jahr 2020 das Global Year für die „Prävention von Schmerzen“ ausgerufen. Mit dieser Kampagne soll das Bewusstsein dafür geschärft werden, dass eine Prävention von Schmerzen und somit von deren Folgen mindestens genauso wichtig ist wie eine Schmerzbehandlung. Wir hatten die einzigartige Gelegenheit, als Vorsitzende der Arbeitsgruppe der IASP-Kampagne (EPZ) und als gewählte Präsidentin der IASP (CS) dieses Jahr zusammen mit einer sehr produktiven Arbeitsgruppe mitgestalten zu dürfen. Inhaltlich sollte ein Überblick über Präventionsstrategien gegeben werden, die dazu in der Lage sind, das Auftreten von Schmerzen zu verhindern (Primärprävention), die Chronifizierung von akuten Schmerzen zu limitieren (Sekundärprävention) und Langzeitfolgen von Schmerzen zu verringern (Tertiärprävention).

Vorbeugende Maßnahmen gegen das Entstehen von Schmerzen oder zum Verhindern ihrer Verstärkung/ Chronifizierung können in jedem Stadium getroffen werden, sind aber erfolgversprechender, je früher sie einsetzen. Dies ist im Schmerzbereich von großer Bedeutung, da einmal chronifizierte Schmerzen eine therapeutische Herausforderung darstellen und der Erfolg von Therapiemaßnahmen dann sehr unterschiedlich und oft nicht zufriedenstellend ist. Allerdings können auch chronische Schmerzen sich durch maladaptive Prozesse verschlechtern; eine Sekundär- und Tertiärprävention ist deshalb von ebenso großer Wichtigkeit wie eine Primärprävention. Die Idee der Umsetzung einer Prävention im Schmerzbereich wird jedoch durch eine Reihe von Faktoren erschwert. So sind z. B. Präventionsstrategien für viele andere chronische Krankheiten gut erforscht und in das Gesundheitswesen integriert, Präventionsstrategien für Schmerzen jedoch häufig nicht. Dies liegt möglicherweise daran, dass besonders gefährdete Risikopatienten frühzeitig identifiziert werden müssen, um ihnen eine angemessene und gezielte Prävention anzubieten. Diese Risikoidentifizierung ist aber vor allem in den früheren Stadien nicht gut etabliert und es fehlen Stratifizierungsmöglichkeiten, um dem Patienten die für ihn maßgeschneiderte Prävention zukommen zu lassen. Bei subakuten Schmerzen mit Risiko zur Chronifizierung besteht oft ein ähnliches Problem; darüber hinaus sind hier Patient und Behandler oft nicht sensibilisiert genug dafür, dass die Schmerzen auch progredient oder rezidivierend sein können und nicht bei jedem Patienten „einfach so" wieder verschwinden. Hier fehlen gezielte „Filtersysteme" zur Identifizierung der Risikopatienten und geeigneter Kurzzeitpräventionsansätze im Gesundheitssystem. In späteren Stadien sind Schmerzpatienten häufig durch Begleitsymptome mit Einschränkungen im alltäglichen Leben zusätzlich beeinträchtigt; eine primäre oder sekundäre Prävention ist dann nicht mehr möglich.
Allerdings wäre zu diesem Zeitpunkt eine Prävention weiterer gesundheitlicher Störungen und von Begleitsymptomen eine Möglichkeit. Es besteht deshalb ein dringender Handlungsbedarf bezüglich der Prävention, in Bezug auf Forschung in diesem Bereich wie auch auf Implementierung dessen, was bereits bekannt ist.

Das Global Year 2020 soll deshalb nicht nur darauf aufmerksam machen, warum Prävention wichtig ist, sondern auch, welche Erkenntnisse bereits vorhanden sind und welche Präventionsstrategien von Angehörigen der Gesundheitsberufe sowie auch von Patienten selber angewendet werden können bzw. wo noch Wissenslücken bestehen. Auf der Website der IASP (https://www. iasp-pain.org/GlobalYear) finden sich zu diesem Thema Factsheets (einschließlich Übersetzungen in mehr als 10 Sprachen, auch ins Deutsche) und Links zu Webinaren mit präventionsbezogenen Themenschwerpunkten. Nicht zuletzt sind im Jahr 2020 auch in den IASPZeitschriften PAIN und PAIN Reports die themenrelevanten Artikel mit dem Logo des Globalen Jahrs identifiziert worden.

In dieser Ausgabe der Zeitschrift Der Schmerz wollen wir nun zusammen mit den Autoren der Beiträge, die alle Experten auf ihrem Gebiet sind, herausstellen, was derzeit über die Primär-, Sekundär- und Tertiärprävention von Schmerzen bekannt und für die deutsche Versorgungslandschaft wichtig ist. 
Hier steht eine Anzeige.

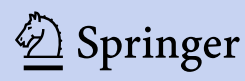


Schwerpunkte hierbei liegen auf der Schmerzprävention durch Übungstherapie und körperliche Aktivität, psychologischen Aspekten, die relevant sind für eine Schmerzprävention, Schmerzprävention in der Grundversorgung (für niedergelassene Ärzte besonders interessant), Prävention chronischer postoperativer Schmerzen und Prävention chronischer Schmerzen durch frühes interdisziplinäres Assessment. Nicht zuletzt wollen wir auch einen Ausblick aus der Forschung geben, was bezüglich der Prävention in den nächsten Jahren Neues kommen könnte. Ein solches Heft kann nicht alle Bereiche abdecken; uns ist deshalb bewusst, dass sicherlich einige Aspekte fehlen, wir hoffen aber hier auf das Verständnis und verweisen auch nochmals auf die vorhandene Website der IASP.

Sowohl die Forschung auf dem Gebiet der Schmerzprävention als auch die Umsetzung der bereits bekannten Möglichkeiten weist noch eine Vielzahl von offenen Fragen auf. Weitere Forschungsarbeiten sind erforderlich, um Präventionsmechanismen, Risikofaktoren und Risikobewertungsinstrumente, präventive Therapieansätze und Responder auf allen Ebenen zu identifizieren, Präventionsansätze in die Klinik zu implementieren, sektorenübergreifende Maßnahmen und frühzeitige Interventionen einzuführen und damit Schmerzentstehung und Schmerzchronifizierung in der $\mathrm{Zu}$ kunft erfolgreich verhindern zu können. Wir würden uns sehr freuen, wenn damit dieses Thema einer breiten Öffentlichkeit zugänglich wird. Die Rolle der Prävention in der Schmerzmedizin muss klarer und besser bekannt werden. Nicht zuletzt wäre es wichtig, Weichen für die Zukunft zu stellen, um Forschung und Praxis auf diesem Gebiet zu stärken.

\section{Korrespondenzadresse}

Univ.-Prof. Dr. med. Esther Pogatzki-Zahn

Klinik für Anästhesiologie, operative

Intensivmedizin und Schmerztherapie,

Universitätsklinikum Münster

Albert-Schweitzer-Campus 1, 48149 Münster,

Deutschland

pogatzki@anit.uni-muenster.de

Prof. Dr. med. Claudia Sommer

Neurologische Klinik, Universität Würzburg

Josef-Schneider-Straße 2, 97080 Würzburg,

Deutschland

sommer@uni-wuerzburg.de

Interessenkonflikt. E. Pogatzki-Zahn hat in den letzten 4 Jahren (2017-2020) im Rahmen von Advisory-Board-Aktivitäten und Vorträgen Berater- und Vortragshonorare von den Firmen Mundipharma und Grünenthal GmbH erhalten. Ihre Forschungstätigkeiten der letzten 3 Jahre sowie aktuell werden von der DFG (PO1319/3-1, PO1319/4-1 und P01319/5-1), dem BMBF, dem IZKF Münster und der "Innovative Medicines Initiative 2 Joint Undertaking" (IMI 2 JU) unterstützt (Grant agreement No. 777500). IMI 2 JU wird durch "European Union's Horizon 2020 research and innovation programme" der Europäischen Union und durch EFPIA unterstützt. C. Sommer erhielt in den Jahren 2017-2020 Beraterhonorare der Firmen Algiax, Alnylam, Air Liquide, Akcea, Astellas, Bayer, Grifols, Takeda und UCB und Vortragshonorare der Firmen Akcea, Alnylam, Merck, Novartis, Pfizer, Sanofi-Aventis und Teva.

\section{Mitgiederbereich der Deutschen Schmerzgesellschaft \\ Bereich für Mitglieder der Deutschen Schmerzgesell- schaft auf SpringerMedizin.de}

Kennen Sie den Bereich für Mitglieder der Deutschen Schmerzgesellschaft auf SpringerMedizin.de?

www.springermedizin.de/dsg/15587528

Hier finden Sie:

- schnellen Zugriff auf die CME-Kurse von Der Schmerz,

- Themensammlungen zu den Deutschen Schmerzkongressen der letzten Jahre,

- in Der Schmerz publizierte Leitlinien,

- das Online-Archiv von Der Schmerz rückwirkend bis 1997,

- die aktuelle Ausgabe als e.Paper zum Offline-Lesen unterwegs.

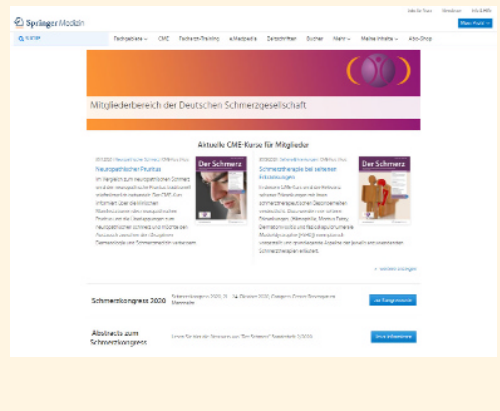

Registrieren Sie sich einmal kostenfrei auf www.springermedizin.de. Geben Sie dabei Vor- und Nachnamen und die Lieferadresse wie auf dem Adressaufkleber auf Ihrem Heft an. So kann im System die Zugehörigkeit zur Deutschen Schmerzgesellschaft sichergestellt werden.

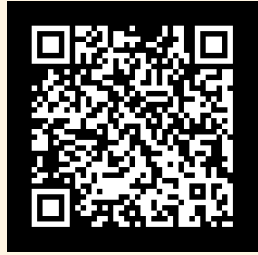

Über diesen QR-Code schnell und einfach lossurfen! 